\title{
Gradhiva
}

GRADHIV

Revue d'anthropologie et d'histoire des arts

13 | 2011

Pièges à voir, pièges à penser

\section{Nubes cum figuris}

L'interprétation des nuages comme paradigme moderne de la perception et de la création artistiques

Nubes cum figuris. The Interpretation of Clouds as a Modern Paradigm of

Artistic Perception and Creation

\section{Dario Gamboni}

Traducteur : Olivier Allard

\section{(2) OpenEdition}

\section{Journals}

Édition électronique

URL : http://journals.openedition.org/gradhiva/2066

DOI : $10.4000 /$ gradhiva.2066

ISSN : 1760-849X

Éditeur

Musée du quai Branly Jacques Chirac

Édition imprimée

Date de publication : 18 mai 2011

Pagination : 148-161

ISBN : 978-2-35744-042-5

ISSN : 0764-8928

Référence électronique

Dario Gamboni, « Nubes cum figuris », Gradhiva [En ligne], 13 | 2011, mis en ligne le 18 mai 2014, consulté le 04 mai 2019. URL : http://journals.openedition.org/gradhiva/2066 ; DOI : 10.4000/ gradhiva.2066

(c) musée du quai Branly 


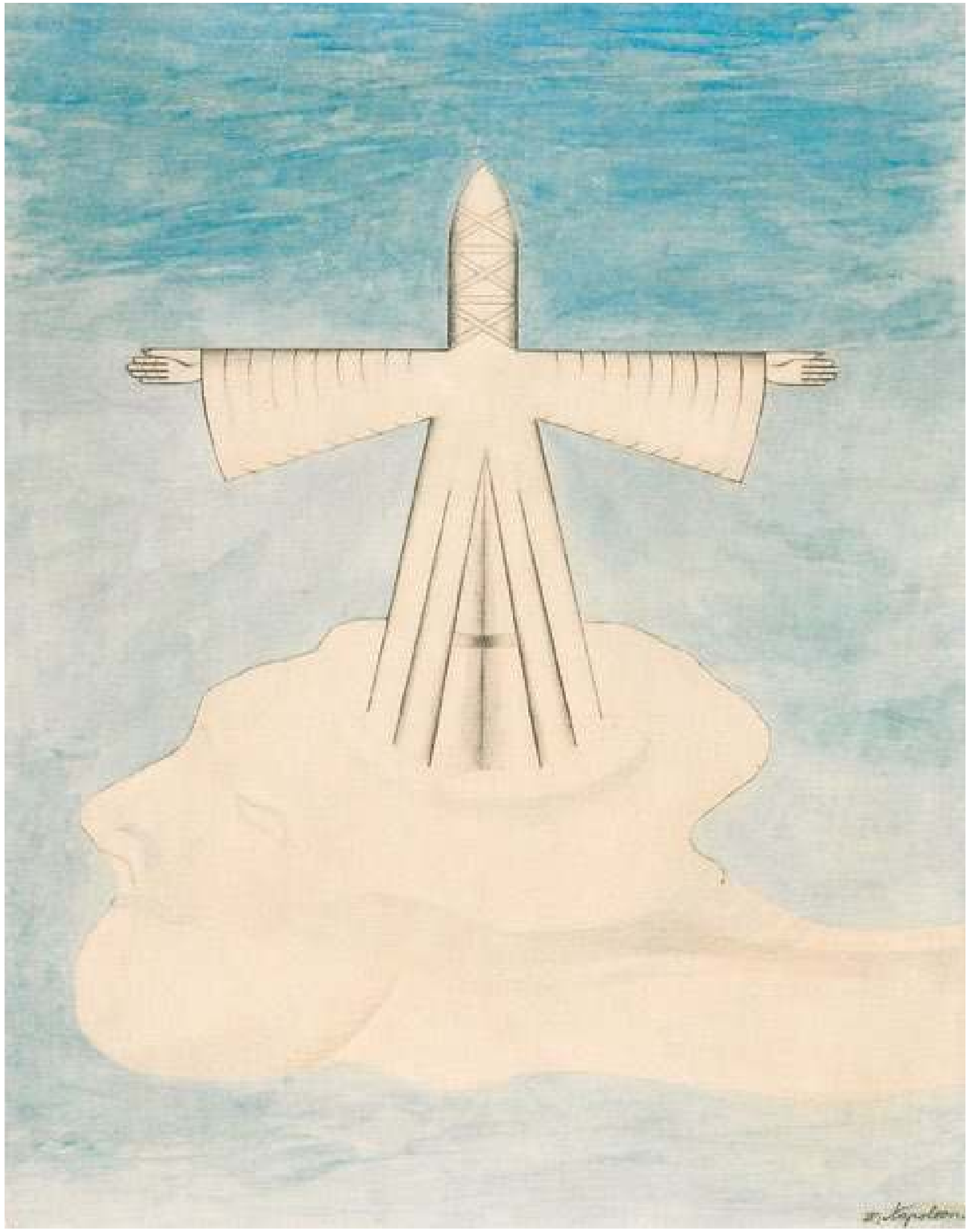

Fig. 1 August Natterer, Antipape, l'ennemi invisible de Dieu dans les nuages, vers 1911-1917, crayon et aquarelle sur papier (26,2 x 20,5 cm). Prinzhorn Collection, Heidelberg. 


\section{Dario Gamboni}

\footnotetext{
- $\circ$

* Ce texte a été publié sous le titre «Nubes cum figuris : The Interpretation of Clouds as a Modern Paradigm of Artistic Perception and Creation », in Alessandro Nova et Tanja Michalsky (dir.), Wind und Wetter. Die Ikonologie der Atmosphäre. Venise, Marsilio, 2009 : 259-266 et 362-372. Traduction de l'anglais par Olivier Allard.
}

1. Gautier 1863 : 1 ?.

2. Philostrate 1862 : 73.

3. Voir Hauser 2001 et Gamboni 2002 : 27-3?.

\section{Nubes cum figuris L'interprétation des nuages
comme paradigme moderne de la
perception et de la création artistiques* L'interprétation des nuages
comme paradigme moderne de la
perception et de la création artistiques* L'interprétation des nuages
comme paradigme moderne de la
perception et de la création artistiques*}

Depuis qu'il y a des nuages et des êtres humains - voire préhumains -, ces derniers y reconnurent probablement des images. Les contours flous et ambigus des nuages en font des "formes faibles" par excellence (au sens de la psychologie de la Gestalt), d'autant plus qu'ils ne cessent de se modifier et de se mouvoir. Le fait qu'ils apparaissent dans le ciel les prédispose à être interprétés comme les images, les travestissements (comme dans le mythe d'Ixion et de Néphélé) ou les messagers d'êtres célestes. Victor Hugo a écrit sur l'un de ses dessins les mots Homo lapides, nubes deus ${ }^{1}$, qui renvoient peut-être à la fragilité des entreprises humaines mais peuvent aussi signifier que Dieu crée des formes avec des nuages comme l'homme en crée avec des pierres. Dès l'Antiquité tardive, l'interprétation des nuages a pu être considérée comme une expression de la dimension imaginative de la perception humaine. Au début du III siècle après Jésus-Christ, Philostrate d'Athènes écrivait que les images vues dans les nuages n'étaient pas des créations divines, mais le produit de l'inclination naturelle des hommes à imiter (mimesis); lorsque l'imitation s'effectuait avec la main et non seulement avec l'esprit, il s'agissait alors d'art ${ }^{2}$. Cette conception réapparut à la Renaissance, par exemple dans les peintures de rochers anthropomorphes par Mantegna, dans les dessins par Dürer de son oreiller suggérant des visages, ou encore lorsque Léonard de Vinci conseilla à ses collègues d'utiliser des murs tachés, des pierres multicolores et des nuages pour développer leur puissance d'invention?3.

Lorsque la nature cessa d'être vue comme un artiste capable de s'imiter luimême, une conception naturaliste de l'imitation artistique marginalisa inévitablement la représentation des images vues dans les nuages. Dans son Intro- 


\section{PIÈGES À VOIR, PIÈGES À PENSER}

\author{
- \\ 4. Hoogstraeten 2006 [1678] : 253 \\ Voir aussi Esmeijer 197 ? \\ 5. Voir Engell 1981. \\ 6. Novalis 1963 : 347 ; De Quincey \\ 1971 [1821] : 106-107, 214-215; \\ Wordsworth 1814 : 90.
}

7. Badt $1960: 20$.

8. Baudelaire 1976 : 627 ; voir ibid. : 666-667 pour l'éloge de Meryon.

9. Baudelaire $1975: 350$. duction à la peinture, le Hollandais Samuel van Hoogstraeten écrivait en 1678 que, bien qu'on puisse voir des choses remarquables dans les nuages : "C'est une sotte illusion populaire d'y croire voir/ Se former quelque animal ou bateau, une illusion qui trompe/ Celui qui ne connaît pas notre art et le berce de chimères./ À ce sujet, un peintre a de meilleurs yeux :/ Il connaît les couleurs, les contours, et également la lumière, / Et juge d'une vue plus précise4. " Cependant, à la fin du xvIII ${ }^{\mathrm{e}}$ siècle, l'imagination commença à être considérée comme une forme supérieure de connaissance et les romantiques donnèrent aux artistes la tâche libératrice de créer comme la nature, plutôt que d'après elles. Novalis mentionnait ainsi les nuages parmi les porteurs de la " grande écriture chiffrée » de la nature, dans laquelle nous voyons "naître d'étranges figures ", et Wordsworth décrivait l'apparition d'une ville colossale dans les nuages, qui servit de modèle aux visions de Thomas De Quincey ${ }^{6}$. Dans son poème Hommage à Howard de 1817, Goethe fit l'éloge de l'analyse scientifique des nuages conduite par Luke Howard, non pas en opposition à celle produite par "notre propre pouvoir imaginatif ", mais par analogie avec cette dernière : l'une comme l'autre « déterminent l'indéterminé », tandis que la divinité prend "plaisir à ces figures changeantes ${ }^{7}$ ». Cette conception des images vues dans les nuages comme des signes naturels et comme les produits de l'imagination humaine survécut à la montée du réalisme, ne serait-ce que de façon marginale. Au milieu du xix ${ }^{\mathrm{e}}$ siècle, Charles Méryon dessina dans le ciel parisien des nuages (fig. 2) dans lesquels, de gauche à droite, des silhouettes surhumaines émergent ou s'évanouissent. Baudelaire fit l'éloge de Méryon dans son Salon de 1859, dans lequel il défendait de manière générale l'artiste "imaginatif » contre l'artiste réaliste ou positiviste qui, selon lui, cherchait à «représenter les choses telles qu'elles sont, ou bien qu'elles seraient, en supposant que [lui-même] n'existe pas $^{8}$ ". Pour le poète, les images vues dans les nuages étaient une manifestation de l'aspiration humaine au divin, comme dans La Soupe et les Nuages, où la contemplation des "merveilleuses constructions de l'impalpable» libère temporairement le narrateur d'une réalité oppressante ${ }^{9}$.

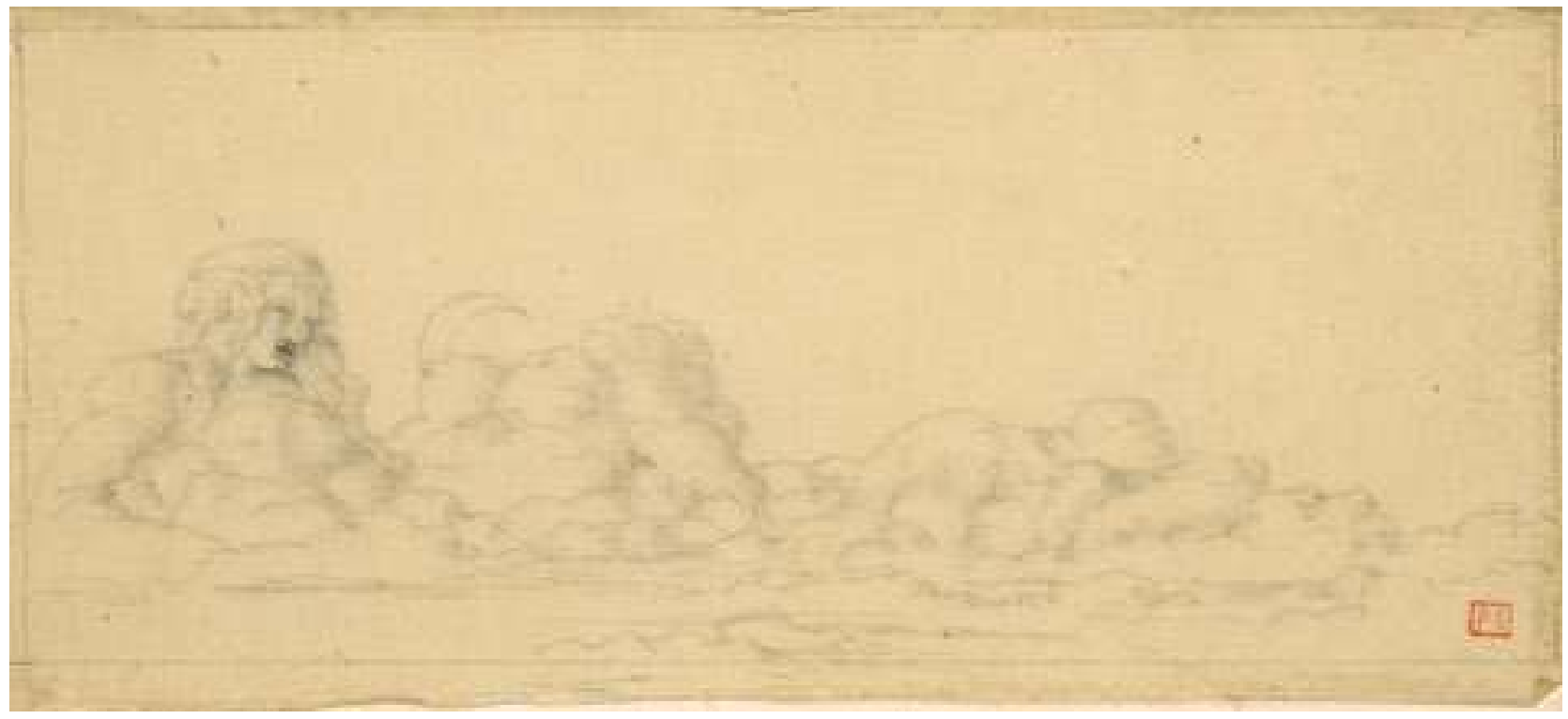

Fig. 2 Charles Méryon, Étude d'un nuage anthropomorphe (deuxième version), vers 1855-1856, crayon sur papier calque ( $9,8 \times 20,2 \mathrm{~cm})$. Sterling and Francine Clark Institute, Williamstown (Mass.) USA/Photo Michael Agee. 


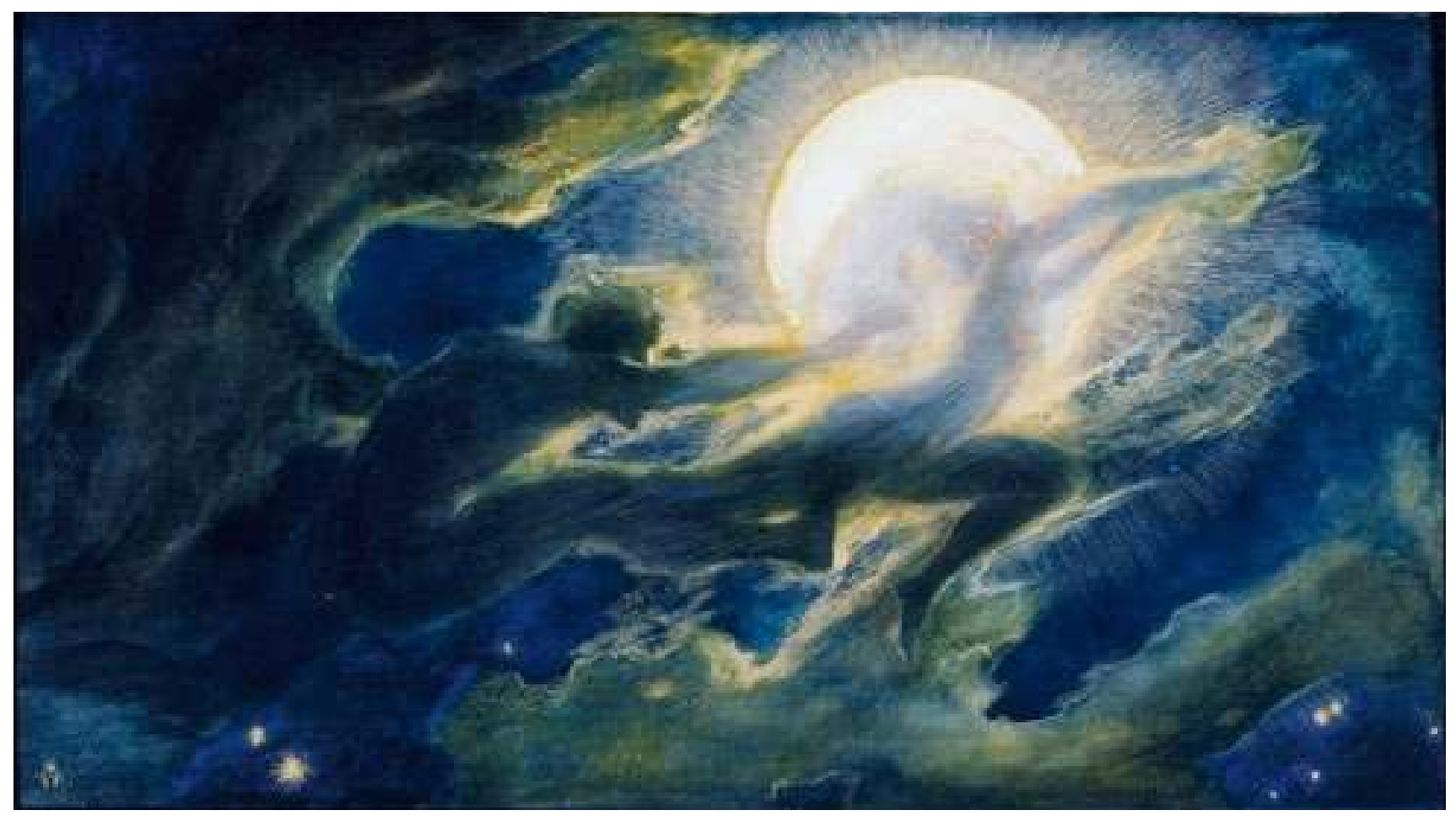

Fig. 3 Richard Riemerschmid, Fantômes dans les nuages, 1897, tempera sur carte $(45 \times 77 \mathrm{~cm})$. Städtische Galerie im Lenbachhaus, Munich.

Baudelaire montrait la voie à une conception devenue prédominante à la fin du xix ${ }^{e}$ siècle et toujours influente de nos jours. Les arts visuels ont perdu et abandonné la tâche de dépeindre l'apparence optique du monde visuel. Concernant les nuages, la photographie - et en particulier la photographie aérienne - était plus apte à représenter l'objectivité extérieure. En 1896 fut publié un atlas international des nuages et le Comité international météorologique déclara 1896-1897 l'« année du nuage ». Des philosophes, des psychologues et des psychophysiologues insistaient sur la dimension active et interprétative de la perception. Hippolyte Taine définit la perception extérieure comme «un rêve du dedans qui se trouve en harmonie avec les choses du dehors", alors qu'Ernst Mach comptait "la projection de phantasmes sur ce qui n'est pas vu clairement" parmi les «manifestations de l'imagination et de la mémoire au sein des perceptions sensorielles ${ }^{10}$ ». Les spécialistes de l'esthétique mobilisaient de telles idées dans leurs analyses de la production et de la réception artistiques : Paul Souriau, par exemple, écrivait en 1893 que " [r] egarder un dessin, c'est voir des chimères dans les nuages ${ }^{11} »$.

Ainsi, la représentation d'images dans les nuages devint plus fréquente, quoique sous des formes très différentes. Le spectateur doit parfois prendre la responsabilité d'interpréter le nuage comme une image, et par conséquent de douter de sa propre «objectivité ». Tina Grütter a récemment suggéré de voir un oiseau dans le nuage impressionnant descendant sur la montagne - qui ressemble ellemême au visage en raccourci du dessin Le Héros mort - dans la peinture tardive La Mort de Giovanni Segantini (1898-1899, musée Segantini, Saint-Moritz) ${ }^{12}$. Les images dans les nuages apparaissent moins ambiguës lorsque, de manière
-

10. Taine 1892 [1870] : 13; Mach 1886 : 83.

11. Souriau 1909 [1893] : 8 ?.

12. Gütter 2004. 
traditionnelle, elles remplissent des fonctions narratives, allégoriques ou commerciales, par exemple dans les Fantômes dans les nuages de Richard Riemerschmid (fig. 3), dans les Nuées de Charles Giron (fig.4), ou dans une publicité pour la compagnie maritime Royal Holland Lloyd où les nuages figurent le lion héraldique. Le terme utilisé par Giron comme titre de son tableau (ainsi que la variante «nue ") a accompagné et suscité de nombreuses interprétations anthropomorphes et souvent érotiques des nuages, en raison de son homophonie avec «nu » (et «nue» au sens de dévêtue). À l'époque, Giron était chargé de décorer le Parlement suisse d'un immense paysage; on trouve dans cette peinture murale, flottant au-dessus du "berceau de la Confédération", des nuages dans lesquels on peut discerner une personnification féminine, de telle sorte que la Suisse est probablement l'un des seuls États où les images dans les nuages apparaissent dans un décor officiel permanent ${ }^{13}$.

Les images dans les nuages possédaient parfois, pour les symbolistes, des dimensions ésotériques ou occultistes: c'est le cas chez August Strindberg, le dramaturge, peintre et photographe suédois, qui décrivit dans son roman autobiographique Inferno (1897) toutes sortes d'images accidentelles, imputées progressivement à des puissances surnaturelles ${ }^{14}$. Mais les artistes symbolistes attribuèrent aussi à de telles images un caractère réflexif, les employant comme un commentaire sur la perception, la représentation et l'art. Dans son aquarelle Les Roches noires (1889, coll. Josefowitz), grâce au vocabulaire formel « abstractodécoratif " caractéristique du synthétisme, Gauguin créa ainsi une continuité entre l'écume et les nuages. Celle-ci devait peut-être évoquer le cycle naturel de l'évaporation et de la pluie, mais elle était aussi probablement destinée à rendre visible le processus humain et artistique de création et de disparition des images, dont un autre exemple est le visage que l'on peut déceler dans le gros rocher noir à gauche. Dans les œuvres plastiques et les écrits d'Odilon Redon, les nuages représentent la nature intrinsèque de la perception, de la création artistique et de la contemplation de l'art. Dans ses premières eaux-fortes déjà (fig. 6), des nuages fantastiques apparaissent au-dessus d'un cavalier solitaire, faisant implicitement allusion au Roi des aulnes de Goethe ${ }^{15}$. En tentant, à l'âge de cinquante ans, de rendre compte de son art, il se souvint comment, pendant son enfance, son père lui avait montré des nuages et demandé s'il y voyait aussi « des apparitions d'êtres bizarres, chimériques et merveilleux ${ }^{16}$ ". À la même époque, il retravailla la plaque de cuivre de sa vieille gravure, la tournant de côté et extrayant ainsi des nuages la chevelure d'une femme nue (fig. 5) - une métamorphose exemplaire, tant du point de vue de l'autobiographie que de la théorie de l'art.

$\mathrm{Au}$ tournant du siècle, le lien entre enfance et perception imaginative fut délicieusement mis en scène par Lyonel Feininger, dans une bande dessinée représentant le monde tel qu'il apparaissait à un enfant, Wee Willy Winkie (fig. 7). Sur une page, ce dernier indique littéralement au dessinateur les formes fantasques que celui-ci doit voir et dépeindre dans les nuages et les falaises. Par une inversion du souvenir d'enfance de Redon et de la relation pédagogique consacrée, c'est ici l'enfant qui instruit l'adulte et l'artiste. Feininger entendait cela sérieusement, comme le révèle sa peinture plus tardive Nuage oiseau (1926, Harvard University Museums, Cambridge, Mass.), un hommage au Moine au bord de la mer de Caspar David Friedrich. On attribuait généralement aux enfants une faculté naturelle, laissée encore indemne par la tradition et l'éducation, également reconnue aux peuples " primitifs» et aux malades mentaux. Le psychiatre allemand Hans Prinzhorn voyait ainsi une "pulsion interprétative " 


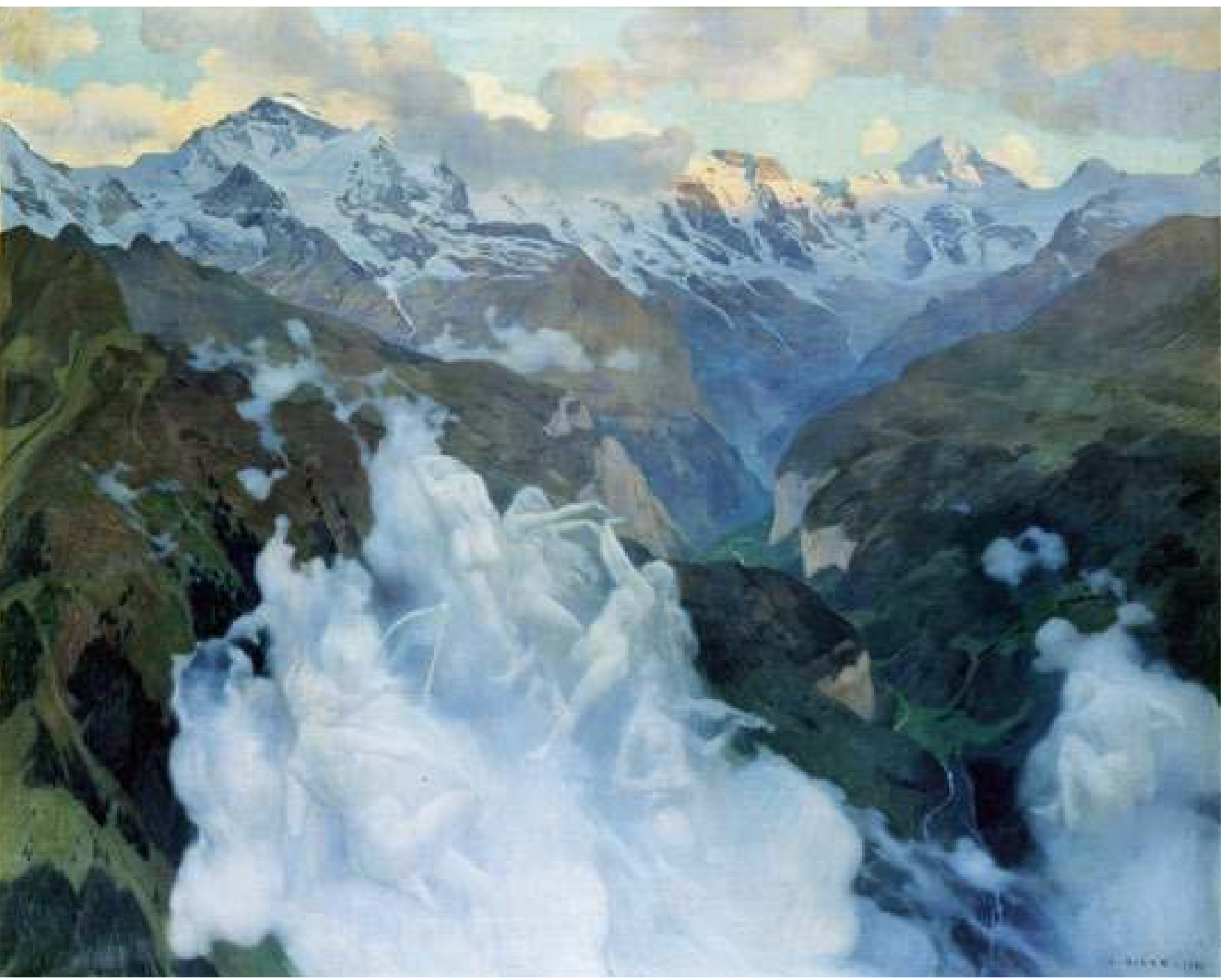

Fig. 4 Charles Giron, Nuées, 1901, huile sur toile $(146,5$ × $181 \mathrm{~cm}) \odot$ musée Jenisch, Vevey/Photo Claude Bornand, Lausanne. 
PIÈGES À VOIR, PIÈGES À PENSER

Fig. 5 Odilon Redon, Baigneuse, 1904, eau-forte et pointe sèche $(13,3 \times 6,3 \mathrm{~cm}), 1920.1524$. The Charles Stickney

Collection $\odot$ The Art Institute of Chicago

Fig. 6 Odilon Redon, Cavalier galopant, 1866, eau-forte et pointe sèche $(6,3 \times 13,5 \mathrm{~cm})$, 1920.1523. The Charles Stickney Collection @ The Art Institute of Chicago
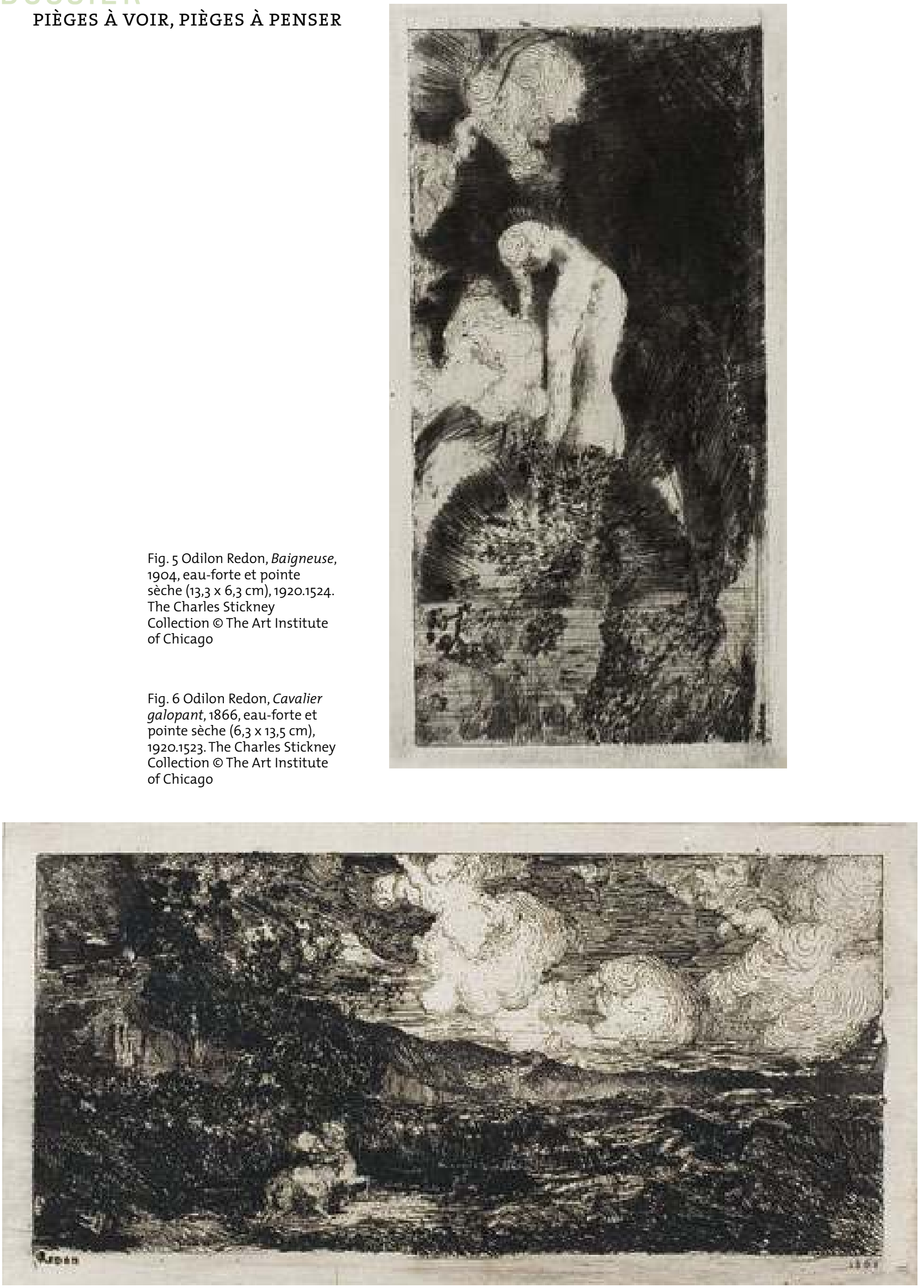


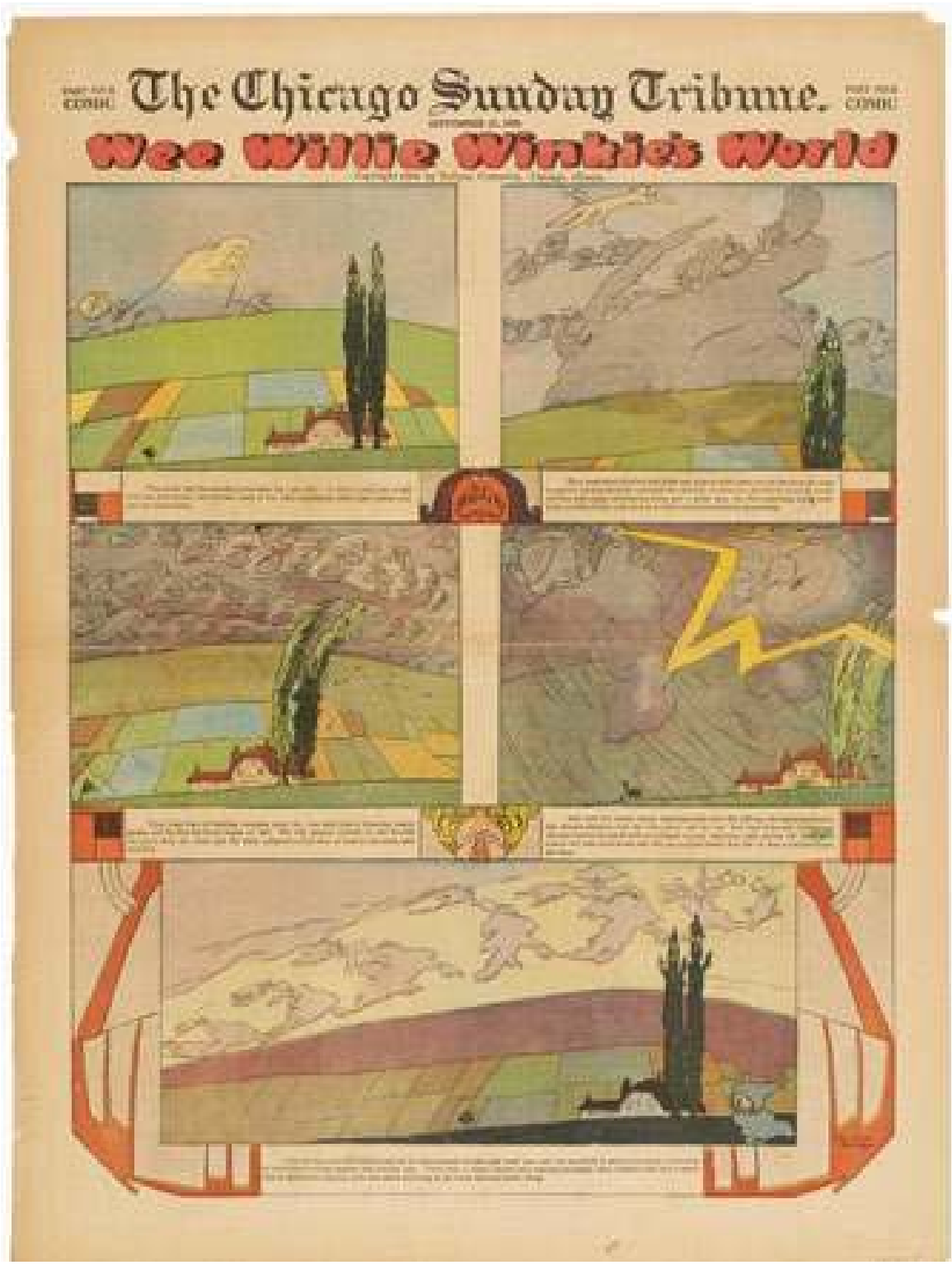

Fig. 7 Lyonel Feininger, planche de la bande dessinée Wee Willie Winkie's World, The Chicago Sunday Tribune, 23 septembre 1906, lithographie $(59,7 \times$ 45,2 cm) @ ADAGP, Paris 2011 @ 2011. Digital Image, The Museum of Modern Art, New York/Scala, Florence.

(Deutungsdrang) à la source de toute activité créatrice, et il comparait l'art préhistorique et populaire avec ce "jeu joué par tous » consistant à regarder «les nuages qui glissent lentement et nous octroient, presque sans effort de notre part, des silhouettes fantastiques ou réalistes à profusion ». Selon lui, analyser les images produites par des malades mentaux offrait les meilleures conditions possibles pour étudier le "processus primaire de création de formes " (primärer Gestaltungsvorgang ${ }^{17}$ ). Dans un des cas les plus importants qu'il présenta, un "système paranoïaque de délire» s'était développé à partir d'une expérience hallucinatoire vue dans les nuages. Les œuvres dérivant de cette expérience (fig. 1) dépeignent les visions apparues à leur auteur dans le ciel, comme si ce dernier représentait un tableau, une toile, une scène ou un écran de cinéma ${ }^{18}$.
- $\bigcirc$

17. Prinzhorn 1994 [1922] : 24-25 et 350-351.

18. Prinzhorn 1994 [1922] : 204 205; Jádi et Brand-Claussen 2001. 
À la même époque, la signification de la "pulsion interprétative " pour la psychologie générale fut mise au premier plan par le psychiatre suisse Hermann Rorschach dans son "expérience diagnostique de perception ", qui devint le plus éminent "test projectif " et un symbole de la psychologie de la perception. Pour Rorschach, l'interprétation n'était qu'un "cas particulier de la perception " et l'interprétation de formes accidentelles constituait une "perception accompagnée par un travail de rapprochement entre sensation et mémoire ${ }^{19}$ \%.

Les surréalistes exaltèrent l'interprétation des formes accidentelles comme automatisme visuel et comme expression de l'inconscient. Dans L'Amour fou, André Breton expliqua la célèbre scène où Hamlet fait trois lectures successives d'un nuage comme une manière de réconcilier la subjectivité et l'objectivité : on devrait conduire sa vie de la même manière qu'on voit dans un nuage ${ }^{20}$. Lorsque, en 1927, Max Ernst raconta son invention de la technique du «frottage ", il eut lui aussi recours à un souvenir d'enfance (fantasmé), dans lequel son père extrayait des silhouettes d'animaux d'un panneau peint "représentant un faux acajou ", à l'aide d'un crayon-fouet évoquant un organe sexuel ${ }^{21}$. Ernst utilisa les veines du bois pour découvrir les images de son Histoire naturelle et y perçut, entre autres choses, un ciel avec la lune (Dans la lumière de la Lune, 1948, coll. part.). En référence à la psychopathologie, Salvador Dalí promut une "pulsion interprétative" intentionnellement exacerbée au rang de "méthode paranoïaque-critique ». Dans son autobiographie, on trouve parmi différents "faux souvenirs d'enfance » un dessin du jeune Salvador et de son père fixant du regard un effrayant nuage de fumée à visage humain, lors d'une promenade à la campa$g \mathrm{e}^{22}$. Des nuages avec des figures apparaissent fréquemment dans son travail, par exemple sur une planche des Chants de Maldoror de 1934 où ils dessinent la silhouette du couple de l'Angelus de Millet, et où le père et le fils peuvent également être remarqués dans le paysage en contrebas.

Entre les deux guerres et jusqu'aux années 1960 - voire, de manière occasionnelle, jusqu'à l'heure actuelle dans la critique d'art -, l'opposition entre «figuration" et "abstraction» (au sens de "non-objectivité) a rendu impossible d'apprécier, de comprendre, voire de percevoir la double mimesis que suppose la représentation d'images dans les nuages. Dans sa conférence de 1968 intitulée "D'autres critères ", Leo Steinberg rejeta à juste titre cette " posture interdictrice - dire à un artiste ce qu'il ne devrait pas faire, et au spectateur ce qu'il ne devrait pas voir ${ }^{23}$ ». La fameuse série de photographies Équivalents (fig. 8), d'Alfred Stieglitz, vues all-over du ciel dépourvues de tout élément d'orientation, en est progressivement venue à être remarquable moins pour son " abstraction " que pour son ambiguïté, qui l'a ainsi rapprochée de l'œuvre d'Edward Weston, dont l'imagination organico-érotique peut facilement être ressentie dans telle photographie d'un nuage en forme de torse (Nuage, Nouveau-Mexique, 1933, coll. part.). 



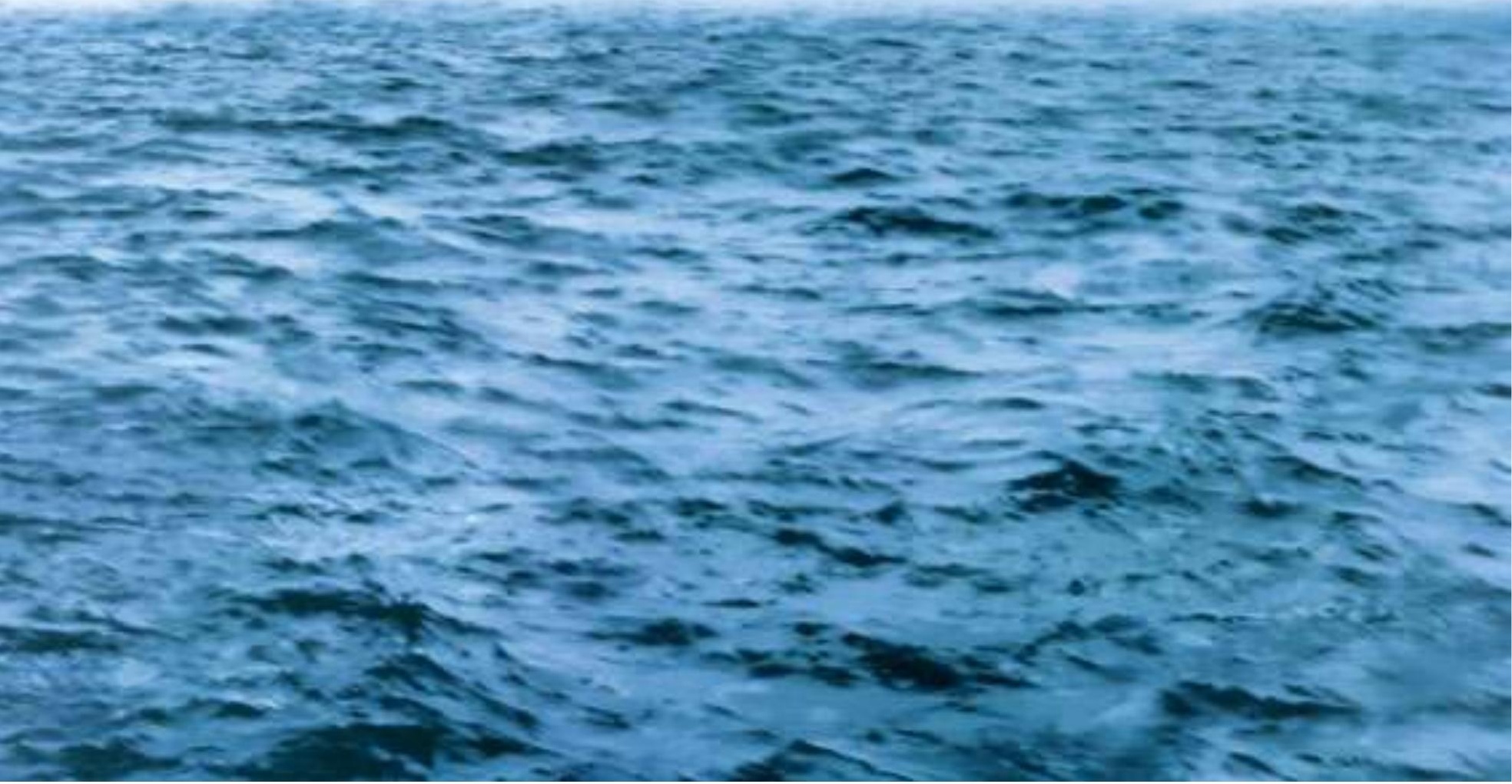

Fig. 9 Gerhard Richter, Marine (Mer-Mer) B 0172, 1970, huile sur toile (200 x 200 cm). Staatliche Museen zu Berlin, Preussischer Kulturbesitz, Nationalgalerie, Berlin @ Gerhard Richter 2011 @ BPK, Berlin, dist. Rmn/Jörg P. Anders. 
Des artistes tels que Sigmar Polke et Gerhard Richter, qui rejetèrent invariablement l'antagonisme entre représentation et abstraction, utilisèrent parfois le motif du ciel et des nuages dans leur réflexion sur la genèse des images. Dans une sorte d'illustration humoristique de la maxime de Breton, Polke découvrit son propre nom dans les constellations ${ }^{24}$. Richter donna forme à l'interchangeabilité d'un ciel nuageux et d'une mer agitée dans sa peinture Marine (Mer-Mer) (fig. 9), dont le sous-titre original (See-See) pourrait être lu comme un jeu de mot anglo-germanique sur "mer" (die See) et "voir" (to see). Ses Peintures abstraites produisent un effet comparable à celui des nuages, du marbre ou du mur taché de Léonard de Vinci, et elles furent clairement réalisées dans cette optique. Faisant écho à la Naturphilosophie romantique et à la conception de l'art de cette dernière, Richter a expliqué que « recourir au hasard revient à peindre comme la Nature » et que, lorsqu'il peint, il est « aveugle comme la Nature, qui agit comme elle peut, au gré des conditions qui l'entravent ou la favorisent ${ }^{25}$ ». Markus Raetz, dont l'œuvre est une étude - par des moyens artistiques - de la théorie de la perception, choisit pour une de ses anamorphoses tridimensionnelles (Not Smoke, 1990, coll. part.) un nuage de fumée s'échappant d'une pipe, qui échange avec celle-ci position et couleur, rendant au passage hommage à Magritte et Foucault ${ }^{26}$. Et dans La Préhistoire des tableaux (1987, Musées de la ville de Strasbourg), Thomas Huber montra cinq nuages, à la structure semblable à du bois veiné, et se déployant au-dessus de la mer comme des colonnes de fumée rejetées par des navires au-delà de l'horizon. Huber voit la surface de l'eau, "point de rencontre entre deux domaines ", comme une image de l'origine des images, puisqu'elles aussi sont « un entre-deux ${ }^{27}$ » ; leur métamorphose ultérieure est suggérée par les nuages vaporeux, comme dans Les Roches noires de Gauguin et dans Marine (Mer-Mer) de Richter.

L'art d'aujourd'hui, dans sa grande diversité, accorde d'autant plus volontiers une place auximages vue dans les nuages que l'ambiguïté et la polysémie y jouent un rôle central. Les films, les vidéos et les images numériques offrent les moyens de réaliser des transformations temporelles et des manipulations potentiellement infinies. Dans les installations vidéo numériques de l'artiste sud-africaine Minette Vári, par exemple, des silhouettes reconnaissables et déformées glissent l'une dans l'autre comme des «nuages se mouvant lentement ». Les images fixes, toutefois, restent capables de créer une tension féconde entre leur propre temporalité et celle du spectateur. Vik Muniz, un producteur de «méta-illusions » tout comme Raetz, s'est attaqué aux Équivalents de Stieglitz à plusieurs occasions ${ }^{28}$. Il a employé ce titre en 1993 pour une série de photographies de morceaux de coton suspendus qui s'apparentent à des nuages ressemblant eux-mêmes - entre autres - à une théière ou à un escargot. Deux ans plus tard, il a participé à l'exposition Le Musée comme muse, au musée d'Art moderne (MoMA) de New York, en présentant un nouvel Équivalent (voir fig. 3 p. 135), une photographie d'une pièce de monnaie peinte en blanc (comme la lune) qu'il avait posée sur le sol en marbre du musée ${ }^{29}$. La fascination exercée par une telle image repose sur le contraste entre son origine particulièrement détournée et réfléchie, d'une part, et son effet stupéfiant et mystérieux, d'autre part. Les peintures murales qu'un manuel de météorologie taoïste a inspirées à l'Américaine Jessica Diamond participent de cette combinaison de sédimentation culturelle et d'apparence spontanée ${ }^{30}$. Le texte comparait les nuages à des orgasmes : à la manière de Polke, un dragon nébuleux projetant du gaz parvient aussi à ressembler au symbole occidental de l'étonnement, un point d'interrogation.

Université de Genève dario.gamboni@unige.ch
PAGE 161

Fig. 10 Veille de rituel tolo. Danse des femmes kuikuro dans le village. Parque Indígena do Xingu, Brésil, 2005. Photo Carlos Fausto.

- $\bigcirc$

24. Sternenhimmeltuch «Sternenhimmel am 24.6. 24 Uhr zeigt als Sternbild den Namenszug S. Polke » (1968, coll. privée), reproduit dans Martin et Andreae (éd.) 2003 : 252.

25 Obrist (éd.) 1993 : 112-113.

26. Voir Martin (éd.) 2009.

27. Huber $1986: 27$ cité dans Huber 1987 : 28

28. Stainback (éd.) 1998: 16. 29. McShine 1999: 134.

30. Patterson 2003. 


\section{Bibliographie}

\section{BADT, Kurt}

1960 Wolkenbilder und Wolkengedichte der Romantik. Berlin, De Gruyter.

\section{Baudelaire, Charles}

1975 CEuvres complètes. Édité par Claude Pichois. T. I. Paris, Gallimard [«Bibliothèque de la Pléiade»].

1976 CEuvres complètes. Édité par Claude Pichois. T. II. Paris, Gallimard [«Bibliothèque de la Pléiade »].

BRETON, André

1997 [1937] L'Amour fou. Paris, Gallimard.

\section{DALí, Salvador}

1993 [1942] The Secret Life of Salvador Dalí. Traduit par Haakon Chevalier. New York, Dover Publication.

\section{De Ouincey, Thomas}

1971 [1821] Confessions of an Opium Eater. Londres, Penguin Books.

ENGELL, James

1981 The Creative Imagination: Enlightenment to Romanticism. Cambridge (Mass.), Harvard University Press.

ERNST, Max

1927 «Trois visions de demi-sommeil », La Révolution surréaliste, 9-10 octobre 1927. 1970 Écritures. Paris, Gallimard.

Esmejuer, Ank C.

1977 «Cloudscapes in Theory and Practice », Simiolus 9 : 123-148.

\section{GAMBOnI, Dario}

2002 Potential Images: Ambiguity and Indeterminacy in Modern Art. London, Reaktion Books.

2004 «Fiction et vérité : un souvenir d'enfance d'Odilon Redon partagé par d'autres artistes », Genesis 24 : 181-18?.

\section{Gautier, Théophile}

1863 Dessins de Victor Hugo, gravés par Paul Chenay, texte par Théophile Gautier. Paris, Castel.

\section{Gavel-Adams, Anne-Charlotte (éd.)} 1994 August Strindberg Samlade Verk. Vol. XXXVII. Stockholm, Norstedts.

\section{GüTTER, Tina}

2004 «Selbstbilniss eines radikalen Pantheisten. Das Gemälde "La Morte" von Giovanni Segantini », Neue Zürcher Zeitung, 8-9 mai 2004 : 65-66.

\section{Hauser, Andreas}

2001 «Andreas Mantegnas "Wolkenreiter". Manifestation von kunstloser Natur oder Ursprung von vexierbildhafter Kunst? 》 in Gerhard von Graevenitz, Stefan Rieger et Felix Thürlemann (éd.), Die Unvermeidlichkeit der Bilder. Tübingen, Gunter Narr Verlag : 147-172.

Hoogstraeten, Samuel van 2006 [1678] Introduction à la haute école de l'art de peinture. Traduit du hollandais par Jan Blanc. Genève, Droz.

\section{Huber, Thomas}

1986 Rede in der Schule. Cologne, Vista Point.

1987 Die Urgerschichte der Bilder/La

Préhistoire des tableaux. Bâle, Museum für Gegenwartskunst.

JÁdI, Inge et Brand-Claussen, Bettina (éd.)

2001 August Natterer. Die Beweiskraft der Bilder. Leben und Werk. Deutungen. Heildelberg, Wunderhorn.

\section{MACH, Ernst}

1886 Beiträge zur Analyse der Empfindungen. Jena, Gustav Fischer.

\section{Martin, Jean-Hubert (éd.)}

2009 Une image peut en cacher une autre. Arcimboldo, Dalí, Raetz. Paris, Réunion des musées nationaux.

\section{Martin, Jean-Hubert et ANDREAE,} Stephan (éd.)

2003 Das endlose Rätsel. Dalí und die Magier der Mehrdeutigkeit. Ostfildern-Ruit, Hatje Cantz Verlag.

\section{McShine, Kynaston (éd.)}

1999 The Museum as Muse: Artists Reflect. New York, Museum of Modern Art.

\section{Novalis}

1963 « Les Disciples à Saïs », traduit par Maurice Maeterlinck, in Maxime Alexandre (éd.), Romantiques allemands. T. I. Paris, Gallimard [«Bibliothèque de la Pléiade »].

\section{OBRIst, Hans Ulrich (éd.)}

1993 Gerhard Richter. Text, Schriften und Interviews. Frankfurt-Leipzig, Insel Verlag.

PAtTerson, Jody

2003 « Jessica Diamond. Montréal Museum of Fine Arts. October 17-January 26 », Parachute $10: 3$.

\section{Philostrate}

1862 Apollonius de Tyane, sa vie, ses voyages, ses prodiges. Traduit par Alexis Chassang.

Paris, Didier.

\section{PRINZHORN, Hans}

1994 [1922] Bildnerei der Geisteskranken. Ein Beitrag zur Psychologie und

Psychopathologie der Gestaltung. VienneNew York, Springer Verlag.

\section{ReDON, Odilon}

1961 [1922] À soi-même. Journal (1867-1915).

Notes sur la vie, l'art et les artistes.

Paris, José Corti.

\section{RoRsCHACH, Hermann}

1948 [1921] Psychodiagnostik.

Methodik und Ergebnisse eines wahrnehmungsdiagnostischen Experiments [Deutenlassen von Zufallsformen].

Berne, Hans Huber.

\section{SoURIAU, Paul}

1909 [1893] La Suggestion dans l'art.

Paris, Alcan.

Stainback, Charles Ashley (éd.)

1998 Vik Muniz: Seeing is Believing. Santa Fé, Arena Editions.

\section{SteinBerg, Leo}

1972 Other Criteria: Confrontations with Twentieth-Century Art. New York, Oxford University Press.

\section{StrindBerg, August}

1898 Inferno. Paris, Mercure de France.

TAIne, Hippolyte

1892 [1870] De l'intelligence. T. II.

Paris, Hachette.

\section{WORDSWORTH, William}

1814 The Excursion. Londres, Longman, Hurst et al. 
